\title{
Accommodation Enhances Depolarizing Inhibition in Central Neurons
}

\author{
Pablo Monsivais and Edwin W Rubel \\ Graduate Program in Neurobiology and Behavior and Virginia Merrill Bloedel Hearing Research Center, University of \\ Washington, Seattle, Washington 98195
}

\begin{abstract}
Neurons in the avian cochlear nucleus are depolarized by GABAergic synaptic input. We recorded GABAergic synaptic currents using the gramicidin-perforated-patch method and found their reversal potential $\left(V_{\text {rev }}\right)$ to be depolarized relative to spike threshold, which is surprising given that these inputs are inhibitory. Depolarizing IPSPs (dIPSPs) are kept below spike generation threshold by the activation of a dendrotoxin-Isensitive, voltage-gated $\mathrm{K}^{+}$conductance. We show experimentally that the polarity of IPSPs contributes to their efficacy; dIPSPs induce accommodation, the positive shift in spike
\end{abstract}

In the CNS, IPSPs typically reduce the excitability of neurons through a combination of two factors: hyperpolarization of the membrane potential away from spike threshold and an increase in the conductance of the cell (Coombs et al., 1955; Smith et al., 1967; Qian and Sejnowski, 1990). Both factors can independently reduce excitability, but in some areas of the nervous system, inhibition is achieved primarily through elevations in membrane conductance that produce no voltage change or even mild depolarization, because the reversal potential for the IPSP is at or slightly above resting potential (Staley and Mody, 1995). This mode of inhibition can be difficult to detect in a quiescent neuron, but the conductance shunts coincident excitatory current, minimizing depolarization.

The depolarization accompanying an inhibitory conductance can itself be an important component of inhibition. In one form of presynaptic inhibition, glycinergic or GABAergic currents depolarize the axon terminal by $10-25 \mathrm{mV}$. Consequently, the amplitude of the presynaptic spike is attenuated through a combination of shunting and $\mathrm{Na}^{+}$channel inactivation. This mechanism of depolarizing inhibition, known as primary afferent depolarization (PAD), has been described in invertebrate sensory fibers, dorsal root ganglion cell terminals, and secretory nerve endings (Eccles et al., 1961; Kennedy et al., 1974; Edwards, 1990; Zhang and Jackson, 1993; Cattaert and El Manira, 1999; Rudomin and Schmidt, 1999). However, in the cell bodies and dendrites of

\footnotetext{
Received April 27, 2001; revised June 19, 2001; accepted July 25, 2001.

This work was supported by National Institutes of Health Grant DC00395, National Institute on Deafness and Other Communication Disorders Grant DC04661, and Institute for General Medical Sciences Training Grant GM07108. We thank M. Binder, H. Brew, D. Perkel, and W. Spain for their comments on earlier versions of this manuscript and J. Gittelman for valuable discussions throughout the study. We are also grateful to M. Binder for access to laboratory equipment and $\mathrm{Y}$. $\mathrm{Lu}$ for help in some of the experiments.

Correspondence should be addressed to Dr. Edwin W Rubel, Graduate Program in Neurobiology and Behavior and Virginia Merrill Bloedel Hearing Research Center, University of Washington, Box 357923, Seattle, WA 98195. E-mail: rubel@u.washington.edu.

Copyright (ㄷ) 2001 Society for Neuroscience $\quad 0270-6474 / 01 / 217823-08 \$ 15.00 / 0$
}

threshold, and are therefore more strongly inhibitory than conventional, hyperpolarizing IPSPs in the same neurons. A similar inhibitory mechanism has been described in invertebrate sensory fibers and axons of dorsal root ganglion cells and may be a general means of amplifying the strength of inhibition in cases where the size of excitatory conductances greatly exceeds that of inhibitory conductances.

Key words: inhibition; GABA; magnocellularis; dynamic clamp; PAD; accommodation; auditory central neurons, such strongly depolarizing GABAergic and glycinergic currents are thought to be rare except during development, when these synaptic inputs are excitatory rather than inhibitory (Owens et al., 1996; Ehrlich et al., 1999).

One place in which depolarizing, GABAergic inputs are functionally inhibitory in mature central neurons is in the avian auditory system. Neurons of nucleus magnocellularis (NM), a division of the cochlear nucleus, exhibit depolarizing, shunting inhibition that is mediated by $\mathrm{GABA}_{\mathrm{A}}$ receptors (Hyson et al., 1995; Monsivais et al., 2000). Intracellular recordings of NM cells in vitro have shown that pressure-applied GABA evokes depolarizations of up to $20 \mathrm{mV}$ (Hyson et al., 1995), but little is known about how depolarization contributes to inhibition in these neurons. The general question of how the polarity of IPSPs influences the efficacy of inhibition has been addressed in modeling studies of presynaptic inhibition (Graham and Redman, 1994). Here, we examined the functional significance of IPSP polarity experimentally, using patch-clamp methods in an in vitro slice preparation.

We made gramicidin-perforated-patch recordings in NM neurons from hatchling chicks and found that the reversal potential of GABAergic IPSPs is depolarized relative to spike threshold by $\sim 10 \mathrm{mV}$. However, depolarizing IPSPs (dIPSPs) are prevented from triggering action potentials by a low-threshold $\mathrm{K}^{+}$conductance that is sensitive to dendrotoxin-I $\left(\mathrm{DTX}_{\mathrm{I}}\right)$, a selective blocker of the KCNA family voltage-gated $\mathrm{K}^{+}$channels. We then mimicked GABAergic input using conductance-clamp recording to evaluate the importance of IPSP polarity on the efficacy of inhibition. We found that dIPSPs provide a more powerful means of reducing excitability than either weakly or strongly hyperpolarizing IPSPs. The functional advantage of depolarizing inhibition relative to hyperpolarizing inhibition was attributable to accommodation, a positive shift in spike threshold. Thus, inactivation of voltage-gated inward currents is likely to play a role in inhibition. The enhancement of inhibitory strength that this mechanism provides may be necessary in NM neurons, which 
receive powerful, calyx-like excitatory inputs from the auditory ganglion.

\section{MATERIALS AND METHODS}

Brainstem slices (150-200 $\mu \mathrm{m}$ thickness) were prepared from day-ofhatch chick embryos and 1-d-old hatchlings as described previously (Reyes et al., 1994; Monsivais et al., 2000). For recording, slices were transferred to a $0.5 \mathrm{cc}$ chamber mounted on a Zeiss (Oberkochen, Germany) Axioskop FS with a $40 \times$ water-immersion objective and infrared, differential interference contrast optics and continuously superfused with artificial CSF (ACSF) at a rate of $3 \mathrm{ml} / \mathrm{min}$. ACSF contained (in mM) $130 \mathrm{NaCl}, 26 \mathrm{NaH}_{2} \mathrm{CO}_{3}, 2.5 \mathrm{KCl}, 2 \mathrm{CaCl}_{2}, 1 \mathrm{MgCl}_{2}, 1.25 \mathrm{NaH}_{2}$ $\mathrm{PO}_{4}$, and 10 dextrose and was constantly gassed with $95 \% \mathrm{O}_{2}$ and $5 \%$ $\mathrm{CO}_{2}$ and had a $\mathrm{pH}$ of 7.4. Unless otherwise noted, all reagents were obtained from Sigma (St. Louis, MO).

During recordings, a slice was perfused with normal ACSF or ACSF containing the AMPA and NMDA glutamate receptor antagonists 6,7dinitroquinoxaline-2,3-dione (DNQX) $(100 \mu \mathrm{M})$ and D,L-2-amino-5phosphonovalerate (APV) $(50 \mu \mathrm{M})$. Dendrotoxin-I (Alomone Labs, Jerusalem, Israel) was used at $0.1 \mu \mathrm{M}$. In some experiments, $50 \mu \mathrm{M}$ bicuculline methiodide was used at the end of the experiment to block GABAergic responses. All recordings were performed at room temperature $\left(22-23^{\circ} \mathrm{C}\right)$.

Patch pipettes were drawn to 1-2 $\mu \mathrm{m}$ tip diameter and had resistances between 3 and $6 \mathrm{M} \Omega$ (DC). In the perforated-patch experiments, two intracellular pipette solutions were used, differing in their $\left[\mathrm{Cl}^{-}\right]$. For NM recordings, a low $\mathrm{Cl}^{-}$internal solution was used $(7 \mathrm{mM})$, so that deterioration or inadvertent rupture of the perforated patch could be detected as a reversal in IPSC polarity at rest. This solution contained (in $\mathrm{mM}$ ): $135 \mathrm{~K}$-gluconate, $5 \mathrm{KCl}, 5 \mathrm{EGTA}, 10 \mathrm{HEPES}$, and $1 \mathrm{MgCl}_{2}$. After filling a pipette tip with this solution, the pipette shank was backfilled by syringe with the same solution containing gramicidin dissolved in DMSO at a final concentration of $25 \mu \mathrm{g} / \mathrm{ml}$. The same approach was used for perforated-patch recordings from other neurons in the brainstem but with an intracellular pipette solution that contained $37 \mathrm{mM} \mathrm{Cl}^{-}$. Because this solution provided a close match for the native $\mathrm{Cl}^{-}$equilibrium of NM cells, it was also used for whole-cell recordings from those neurons. This solution contained (in mM): $105 \mathrm{~K}$-gluconate, $35 \mathrm{KCl}$, 5 EGTA, 10 $\mathrm{K}-\mathrm{HEPES}$, and $1 \mathrm{MgCl}_{2}$. The $\mathrm{pH}$ of the both solutions was adjusted to 7.2 with $\mathrm{KOH}$, and osmolarity was measured between 280 and 290 mOsm. The liquid junction potentials (measured in ACSF) were $10 \mathrm{mV}$ for the $7 \mathrm{mM} \mathrm{Cl}^{-}$solution and $7 \mathrm{mV}$ for the higher $\mathrm{Cl}^{-}$solution. All data are presented with correction for these junction potentials.

Perforated-patch and whole-cell voltage-clamp recordings were made with the Axopatch 200B amplifier, whereas whole-cell current and conductance-clamp recordings were made with the Axoclamp 2B (Axon Instruments, Union City, CA). For perforated-patch recording, initial series resistance measurements exceeded $100 \mathrm{M} \Omega$ after gigaohm seal formation but could decline to $<20 \mathrm{M} \Omega$ within $20-45$ min, at which time data acquisition began. In each recording, series resistance was compensated by $80-85 \%$, and the voltage errors induced by the uncompensated resistance were corrected offline. Recordings were aborted if the perforated patch ruptured, which was easily detected by an abrupt drop in series resistance and a reversal of IPSC polarity. Data were low-pass filtered at $10 \mathrm{kHz}$ and digitized with an ITC-16 (Instrutech, Great Neck, NY) at $20 \mathrm{kHz}$ for both online and offline analysis. All recording protocols were written and run using the acquisition and analysis software Axograph, version 4.3 (Axon Instruments).

Extracellular stimuli were delivered via a bipolar tungsten electrode positioned in the fiber tract that contains axons from the superior olivary nucleus (SON) (Monsivais et al., 2000). Square electric pulses of 100 $\mu \mathrm{sec}$ duration were delivered through a stimulus isolation unit at intensities ranging from 2 to $40 \mathrm{~V}$. For neurons in the reticular formation, stimuli were delivered and synaptic currents were averaged (at least 10 trials per average) and measured at their peak. In NM recordings, responses to single stimuli were difficult to detect above the baseline noise level when cells were being held at depolarized membrane potentials (see Fig. 1, top left trace). We therefore used trains of stimuli to enhance the synaptic conductance $(10$ stimuli at $100 \mathrm{~Hz})$ and measured current at $5 \mathrm{msec}$ after the last stimulus of a train. We examined synaptic potentials in current clamp, taking care to keep the membrane potential within $5 \mathrm{mV}$ of $-67 \mathrm{mV}$. This was especially important in experiments involving dendrotoxin, because this agent caused mild depolarization in two of four recordings. Neurons with membrane potentials higher or lower were adjusted with DC current. GABAergic currents in SON neurons were evoked by pressure application of GABA (50 mM) dissolved in ACSF. Pressure pulses were between 4 and 10 psi and 10-50 msec in duration and presented once at each holding potential.

Conductance-clamp experiments were performed using a custommade (electronics shop, University of Washington Department of Physiology and Biophysics), analog multiplying amplifier that applied current $\left(I_{\text {IPSP }}\right)$ dynamically $(120 \mathrm{kHz}$ response frequency) to the neuron as a function of conductance $\left(g_{\mathrm{IPSP}}\right)$ and synaptic driving force: $I_{\mathrm{IPSP}}=$ $g_{\text {IPSP }}\left(V_{\text {membrane }}-V_{\text {IPSP }}\right)$.

The synaptic conductance, $g_{\text {IPSP }}$, had a rising phase described by a single exponential with $\tau$ of $1 \mathrm{msec}$, whereas the decaying phase was made up of two exponentials: $\tau_{\text {fast }}$ of $3 \mathrm{msec}$ and $\tau_{\text {slow }}$ of $50 \mathrm{msec}$. The reversal potential of the synaptic current $\left(V_{\text {IPSP }}\right)$ was varied experimentally. The kinetics of the conductance waveform were based on measurements of single IPSCs recorded under perforated-patch voltage clamp from a representative $\mathrm{NM}$ neuron clamped to $-57 \mathrm{mV}$ (see Fig. $4 A$ ). We determined the mimicking of the amplifier of $g_{\text {IPSP }}$ and $V_{\text {IPSP }}$ empirically by applying conductance steps to an artificial cell (resistor-capacitor circuit), and plotting $I-V$ relationships for the steady-state voltage and current (data not shown). These plots assured us of the linearity with which the current output of the amplifier was modulated with changes in voltage (i.e., the linearity of the artificial conductance) and allowed us to confirm the different reversal potentials that were tested in this study. The integration of IPSPs and excitatory stimuli was examined by pairing IPSPs with $2 \mathrm{msec}$ duration pulses of depolarizing injected current (generated by the current-passing circuitry of the Axoclamp amplifier).

\section{RESULTS}

\section{Reversal potential of GABAergic currents exceeds spike threshold}

To record GABAergic currents without disrupting the normal intracellular $\mathrm{Cl}^{-}$concentration, we made gramicidin-perforatedpatch recordings from the somata of visually identified neurons in NM and other areas of the brainstem (Kyrozis and Reichling, 1995). GABAergic currents were recorded in the presence of the glutamate receptor antagonists DNQX and APV, and at the end of some recordings, were blocked by bicuculline to confirm their pharmacological identity.

We first examined spontaneous GABAergic currents in NM under voltage clamp $(n=3)$ and found that they were inward over a range of membrane potentials as positive as $-40 \mathrm{mV}$ (Fig. 1, left). Because the whole-cell noise level increased with greater depolarization, we could not reliably identify a reversal potential for these spontaneous currents. In some recordings, we verified the integrity of the gramicidin-perforated patch by intentionally rupturing it with a brief pulse of negative pressure, leading to dialysis of the neuron with the $7 \mathrm{mM} \mathrm{Cl}^{-}$pipette solution and a change in the reversal potential of GABAergic currents (Fig. 1, right).

Next, we compared the reversal potential $\left(V_{\text {rev }}\right)$ of evoked GABAergic currents in NM cells relative to other neurons in the brainstem. Figure $2 A$ shows an example of GABAergic currents in a NM neuron evoked with a train of stimuli applied to the afferent axons $(10$ shocks at $100 \mathrm{~Hz})$. Currents were inward when the membrane potential was clamped to approximately resting potential $(-61 \mathrm{mV})$ and also when depolarized to $-42 \mathrm{mV}$ but were outward at $-14 \mathrm{mV}$. In contrast, other neurons in the brainstem show GABAergic currents that are outward near resting potential (Fig. 2B). The $V_{\text {rev }}$ in this reticular formation neuron was shifted to a more depolarized level when the perforated patch was ruptured (pipette $\left[\mathrm{Cl}^{-}\right]$of $37 \mathrm{~mm}$ ) (Fig. $2 \mathrm{~B}$, inset).

The current-voltage relationships for these example recordings are shown in Figure $2 C$. Least-squares regression of the data show that GABAergic currents reverse at $-37 \mathrm{mV}$ for the $\mathrm{NM}$ cell in Figure $2 A$ and at -65 for the reticular formation neuron in 


\section{perforated-patch}

\section{whole cell \\ $(7 \mathrm{mM} \mathrm{Cl})$}

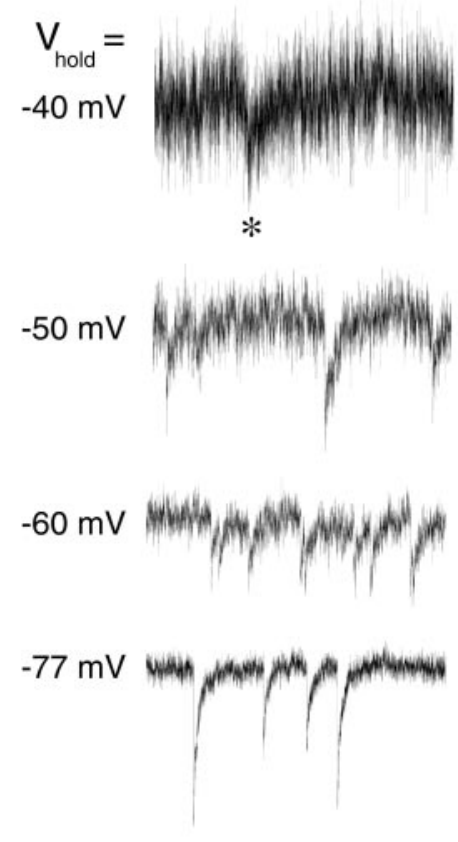

$-85 \mathrm{mV}$

Figure 1. Spontaneous GABAergic currents recorded under gramicidinperforated-patch voltage clamp. The left panel shows that these synaptic currents are inward at holding potentials as depolarized as $-40 \mathrm{mV}$ (asterisk indicates synaptic event). The right panel shows rupture of the patch during the same recording, allowing cell dialysis with the pipette solution $\left(7 \mathrm{mM} \mathrm{Cl}^{-}\right)$, causing a shift in the polarity of currents near rest $(-60 \mathrm{mV})$. The GABAergic currents in the whole-cell recording reversed again at a holding potential of $-85 \mathrm{mV}$, consistent with the predicted whole-cell reversal potential of $-75 \mathrm{mV}$ (note that superfusate contains DNQX and APV).

Figure $2 B$. The average $V_{\text {rev }}$ for GABAergic currents in NM neurons was $-36 \pm 2.1 \mathrm{mV}$ (mean $\pm \mathrm{SEM} ; n=6$ ). In contrast, neurons in another auditory nucleus in the brainstem, the SON, had an average $V_{\text {rev }}$ for GABAergic currents of $-61 \pm 4.3 \mathrm{mV}$ $(n=3)$. Neurons in the reticular formation were measured $(n=$ 6) for an average $V_{\text {rev }}$ of $-72 \pm 4.7 \mathrm{mV}$ (Fig. $2 D$ ).

\section{Low-threshold $\mathrm{K}^{+}$currents limit the depolarization induced by GABAergic IPSPs}

Although the $V_{\text {rev }}$ value we obtained in our NM recordings exceeded spike threshold $(-45 \pm 1.8 \mathrm{mV} ; n=10$; method described below), GABAergic PSPs did not trigger action potentials, in part because of the activation of low-threshold $\mathrm{K}^{+}$currents. Under current clamp, we made whole-cell recordings using physiologically realistic intracellular $\left[\mathrm{Cl}^{-}\right]\left(37 \mathrm{~mm}\right.$; predicted $V_{\mathrm{rev}}$ of $-34 \mathrm{mV}$ ) to investigate the role of voltage-gated $\mathrm{K}^{+}$conductances in shaping and limiting the depolarizations caused by GABAergic input. Under control conditions, dIPSPs reached peak amplitudes of $10-12 \mathrm{mV}$ above rest and decayed biphasically (Fig. 3A, arrow).

In the presence of bath-applied $\mathrm{DTX}_{\mathrm{I}}$, a $\mathrm{K}^{+}$channel toxin selective for KCNA (Kv-1.x) subunits 1,2 , and possibly 6 (Robertson and Owen, 1992; Hopkins et al., 1994), the same stimulus intensity produced dIPSPs that were excitatory, triggering single or brief bursts of spikes $(n=4)$ (Fig. $3 B)$. Also, subthreshold potentials were kinetically different from control IPSPs, decaying smoothly instead of biphasically (arrowhead). Although DTX may have had presynaptic effects, its postsynaptic effects were sufficient to account for the ability of dIPSPs to evoke spikes.

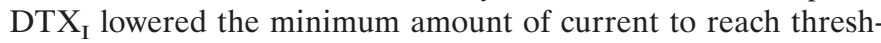
old and enabled repetitive firing in response to steps of direct current in NM neurons ( $n=3$; data not shown), findings that are consistent with the block of low-threshold $\mathrm{K}^{+}$currents (Reyes et al., 1994; Rathouz and Trussell, 1998). Similarly, spontaneous

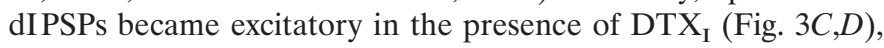
and subthreshold PSPs in DTX differed kinetically from control

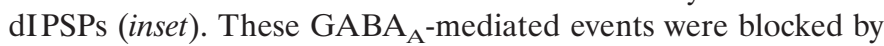
bicuculline (Fig. 3E).

\section{Properties of IPSPs mimicked under conductance clamp}

To investigate how the polarity of IPSPs influences the strength of inhibition, we mimicked GABAergic potentials in NM neurons under dynamic clamp (Robinson and Kawai, 1993; Sharp et al., 1993) $(n=10)$. This approach allowed us to examine the integration of IPSPs evoked by conductance changes of known amplitudes and let us compare the strength of positive and negative polarity IPSPs by varying the reversal potential of the mimicked synaptic current. The conductance waveform was taken from stimulus-evoked IPSCs that had been recorded under perforatedpatch voltage clamp and was represented by a triple-exponential waveform (Fig. 4A) (see Materials and Methods). This waveform was used in all cells, and only the peak amplitude of the conductance was varied among cells: $7.5(n=7)$ and $10(n=3) \mathrm{nS}$. These values are within the range for inhibitory synaptic conductances measured in our voltage-clamp experiments $(10.5 \pm 2.7 \mathrm{nS} ; n=$ 5) and produced dIPSPs with amplitudes ranging from 5 to $12 \mathrm{mV}$ above resting potential when $V_{\text {rev }}$ was set to $-37 \mathrm{mV}$. This range is similar to what we have reported from whole-cell recordings of stimulus-evoked dIPSPs using a physiologically realistic internal $\mathrm{Cl}^{-}$concentration (Monsivais et al., 2000).

Figure $4 B$ illustrates the mimicked GABAergic conductance stimulus (top trace; peak conductance of $10 \mathrm{nS}$ ) and a family of injected currents and voltage responses recorded under dynamic clamp (bottom traces). Currents and voltages corresponding to each of three reversal potentials are shown: $-37,-77$, and -87 $\mathrm{mV}$. Like their natural counterparts, mimicked dIPSPs activated low-threshold $\mathrm{K}^{+}$currents, speeding their repolarization and producing a rapid component to the decaying phase. Hyperpolarizing IPSPs also decayed biphasically in most cells, presumably because of the rapid deactivation of standing outward currents (Rathouz and Trussell, 1998).

The shape of mimicked IPSPs could be changed from biphasically decaying to smoothly decaying by hyperpolarizing the baseline membrane potential and thereby preventing the activation of low-threshold $\mathrm{K}^{+}$conductances (Fig. 4C). Mimicked dIPSPs were kinetically similar to spontaneous and evoked dIPSPs. The similarity is illustrated for one neuron by superimposing a mimicked dIPSP on a spontaneous dIPSP of similar amplitude (Fig. 4D).

\section{Depolarizing IPSPs inhibit firing more effectively than hyperpolarizing IPSPs}

We measured IPSP-induced changes in excitability by injecting paired pulses ( $2 \mathrm{msec}$ duration, $20 \mathrm{msec}$ interpulse interval) of direct current at a range of amplitudes, with the second pulse beginning $8 \mathrm{msec}$ after the onset of the synaptic conductance. The 
A

NM neuron

Figure 2. Reversal potential of GABAergic currents in NM neurons is more positive than that found in other neurons. $A$, Gramicidinperforated-patch voltage-clamp recordings of GABAergic currents evoked by synaptic stimulation. Membrane potential was held at different voltages, and average current amplitude (10 trials) was measured $5 \mathrm{msec}$ after the last stimulus (outlined by vertical bar). B, Gramicidin-perforated-patch recordings from a reticular formation neuron in a slice from the same animal show that GABAergic currents reverse at a more hyperpolarized membrane potential. Traces shown are recorded at holding potentials of $-91,-76,-68,-59$, and $-49 \mathrm{mV}$. Inset shows that the reversal potential changes to more a positive value after patch rupture ([Cl- $\left.{ }^{-}\right]$pipette of $\left.37 \mathrm{~mm}\right)$. $C$, NM data ( $d i-$ amonds) fit with a linear regression gives a $V_{\text {rev }}$ of $-37 \mathrm{mV}$, whereas GABAergic currents in a brainstem neuron (circles) show a more hyperpolarized $V_{\text {rev }}$ of approximately $-65 \mathrm{mV}$. $D$, Mean \pm SEM $V_{\text {rev }}$ for GABAergic currents in NM neurons was $-36 \pm 2.1 \mathrm{mV}$. Auditory neurons in the SON and other brainstem neurons had more hyperpolarized $V_{\text {rev }}$ averages of $-61 \pm 4.3$ and $-72 \pm 4.7$, respectively (note that currents recorded in the presence of DNQX and APV).
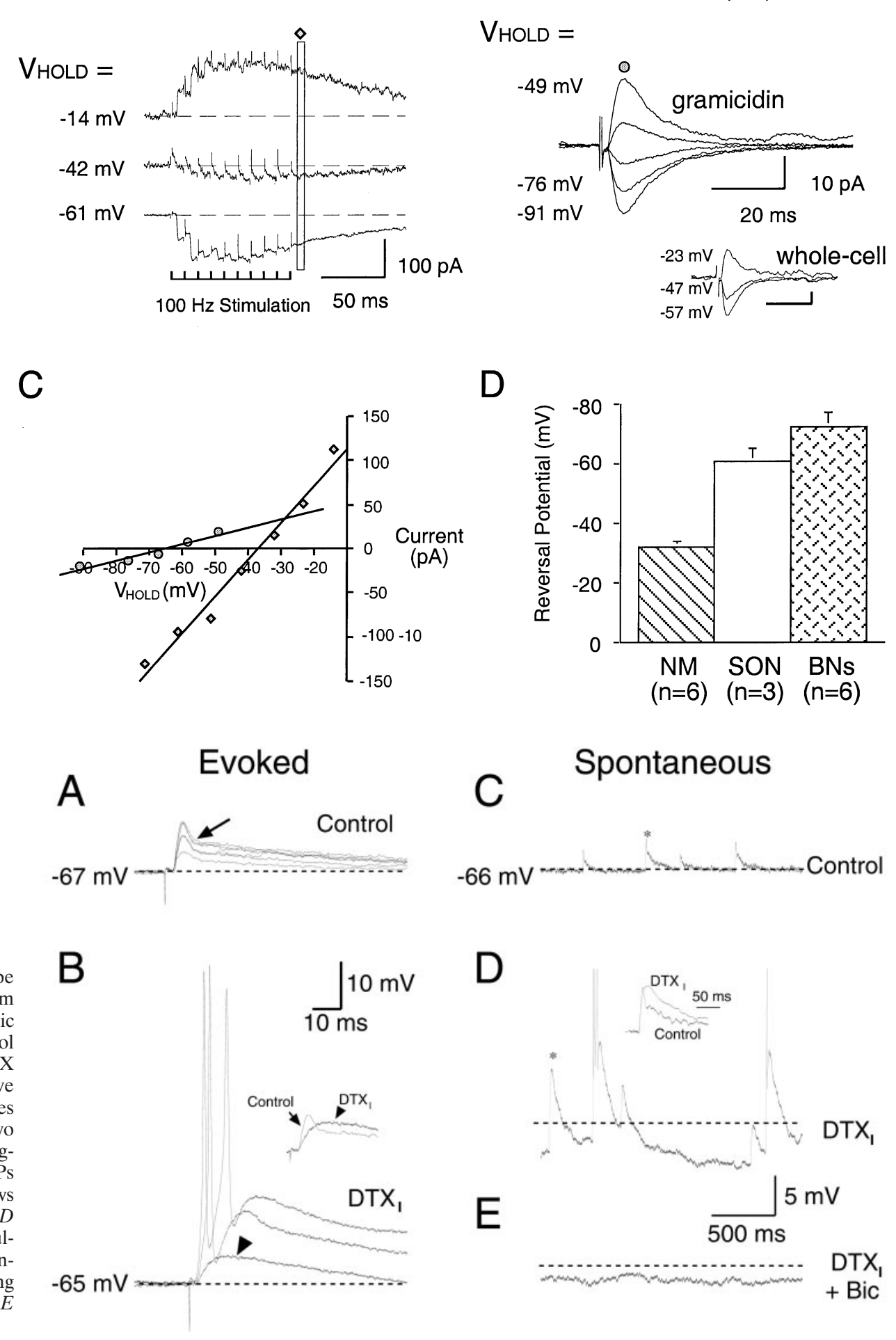

B

Brainstem neuron

(BN)

\section{Spontaneous}

The inhibition was attributable to an increase in the minimum amount of current that was required to reach threshold, or threshold current. We quantified inhibitory efficacy of IPSPs across neurons by measuring the amount of additional current that was required to elicit $40-60 \%$ firing probability during the IPSP relative to when the cell was at rest. Both depolarizing and hyperpolarizing IPSPs reduced excitability. However, dIPSPs
Figure 3. Low-threshold $\mathrm{K}^{+}$currents shape depolarizing IPSPs and prevent them from triggering spikes. Stimulus-evoked GABAergic IPSPs recorded in current clamp under control conditions $(A)$ (superfusate contains DNQX and APV) and in the presence of the selective $\mathrm{K}^{+}$channel blocker DTX $\mathrm{I}_{\mathrm{I}}(B)$. Inset illustrates the difference in IPSP shape between the two conditions. $C$, Spontaneous IPSPs are also augmented in $\operatorname{DTX}_{\mathrm{I}}(D)$, with the largest IPSPs triggering spikes (truncated). Inset in $D$ shows amplitude-normalized dIPSPs from $\mathrm{C}$ and $D$ (asterisk indicates marked events). E, Bicuculline $(50 \mu \mathrm{M})$ completely abolishes these spontaneous potentials. Note that hyperpolarizing DC current $(-20 \mathrm{pA})$ was applied in $D$ and $E$ to minimize spontaneous firing. stimulus paradigm is illustrated in Figure $5 A$. With a conductance peak of $7.5 \mathrm{nS}$ and $V_{\text {rev }}$ of $-37 \mathrm{mV}$, the resulting depolarizing IPSPs inhibited action potentials evoked by $800 \mathrm{pA}$ current pulses in 10 of 10 trials (Fig. 5B, top). In contrast, weakly hyperpolarizing IPSPs $\left(V_{\text {rev }}\right.$ of $-77 \mathrm{mV}$ ) (Fig. $5 B$, middle) or strongly hyperpolarizing IPSPs ( $V_{\text {rev }}$ of $-87 \mathrm{mV}$ ) (Fig. $5 B$, bottom) failed to inhibit spike generation. 


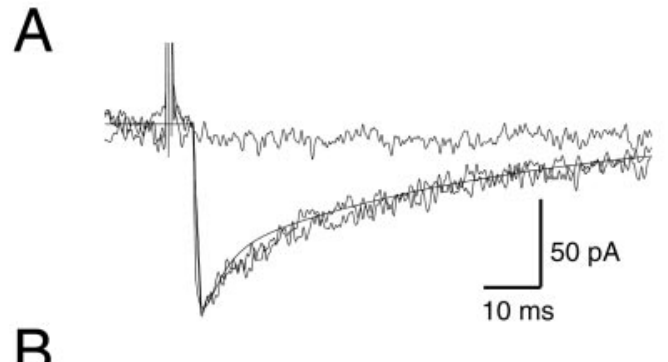

B
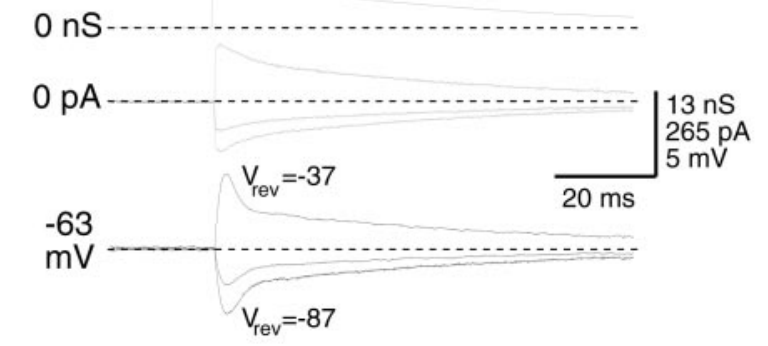

C

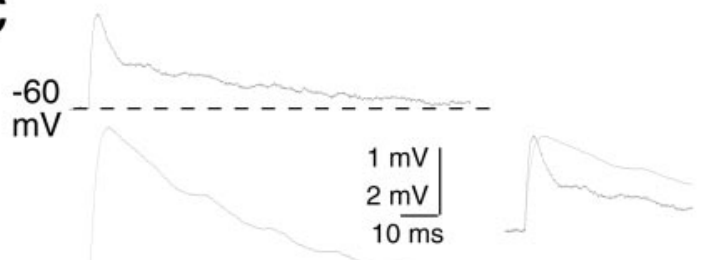

$-92$

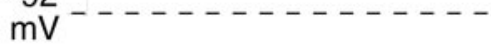

D

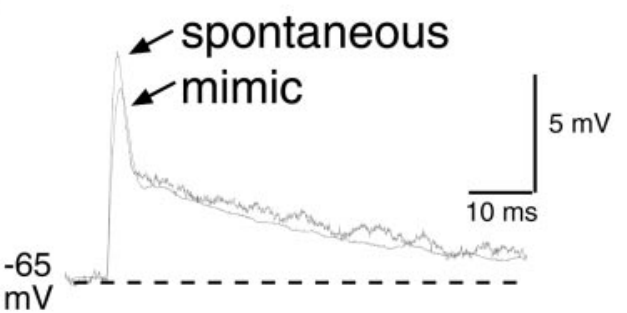

Figure 4. Properties of mimicked IPSPs under conductance clamp. $A$, Evoked GABAergic synaptic currents recorded in a representative NM neuron ( 1 failure in 3 trials) and a superimposed conductance waveform made up three exponentials (see Materials and Methods). $B$, The GABAergic conductance waveform with conductance peak $\left(g_{\text {peak }}\right)$ of 10 $\mathrm{nS}$ (top trace) and a family of currents and voltages corresponding to three different reversal potentials: $-37,-77$, and $-87 \mathrm{mV}$. Note that the dashed line in each trace indicates baseline level. $C$, In another cell, biphasic decay of depolarizing IPSP ( $g_{\text {peak }}$ of 7.5; $V_{\text {rev }}$ of -37$)$ is eliminated by hyperpolarization of resting baseline membrane potential (bottom trace). Note the difference in vertical scale: $1 \mathrm{mV}$ for the top trace and $2 \mathrm{mV}$ for the bottom trace. Inset shows amplitude-normalized traces for easier comparison (time scale unchanged). $D$, Mimicked IPSPs are kinetically similar to spontaneous and evoked IPSPs, as shown in another cell by overlaying an average of 10 mimicked IPSPs ( $V_{\text {rev }}$ of $-37 ; g_{\text {peak }}$ of $\left.7.5 \mathrm{nS}\right)$ with a single, spontaneous IPSP of similar peak amplitude.

inhibited more robustly than hyperpolarizing IPSPs; threshold current during dIPSPs increased to an average of $156 \%$ of control, whereas IPSPs with reversal potentials of -77 and $-87 \mathrm{mV}$ increased average threshold current to 125 and $124 \%$ of control, respectively $(n=10)$ (Fig. $5 C)$. Because the two excitatory stimuli occurred with an interval of $20 \mathrm{msec}$, a relative refractoriness

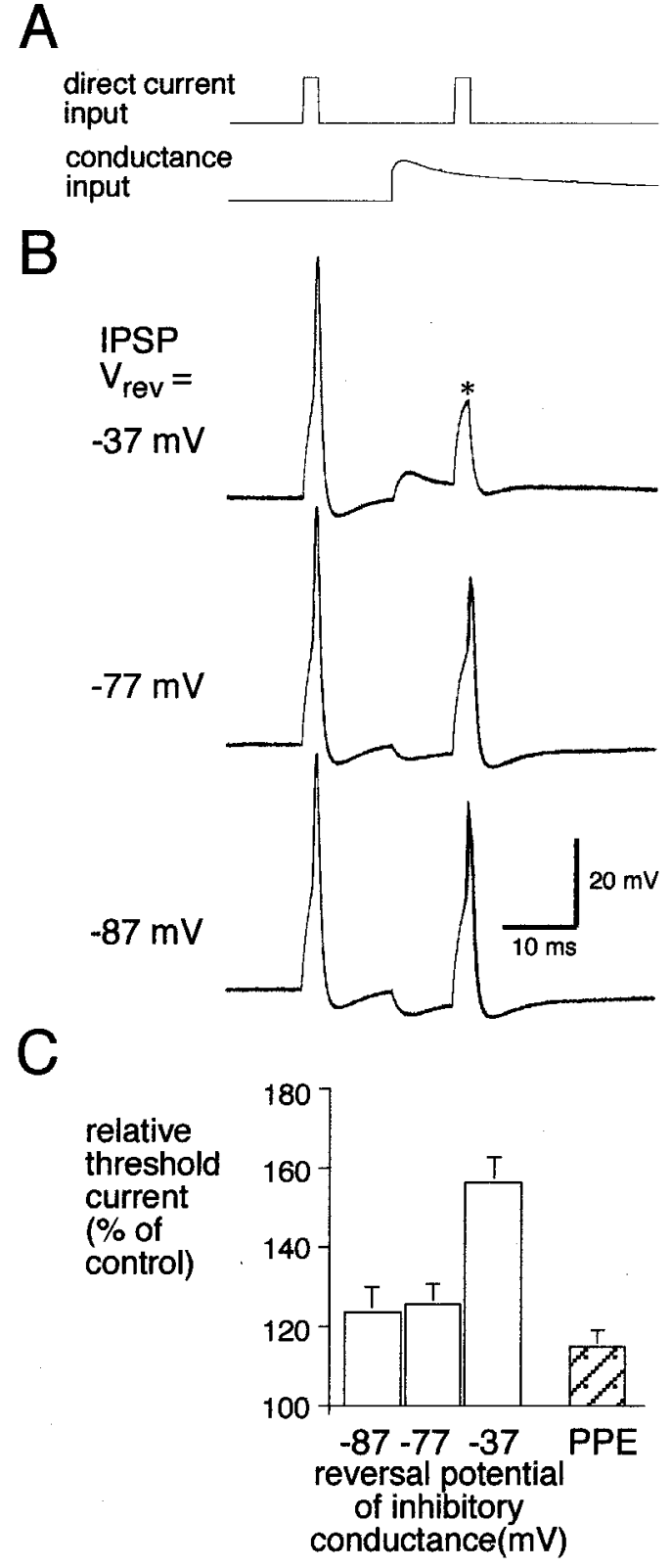

Figure 5. Depolarizing IPSPs inhibit more effectively than hyperpolarizing IPSPs. $A$, The paradigm used for evaluating the excitability of neurons during mimicked IPSPs in conductance clamp. Two suprathreshold current pulses were injected, the second coinciding with the decaying phase of the mimicked IPSP. $B$, Depolarizing inhibition suppressed firing in 10 of 10 trials (top trace; asterisk), whereas weakly hyperpolarizing IPSPs (middle trace) or strongly hyperpolarizing IPSPs (bottom trace) did not. $C$, Data for 10 neurons (same protocol as in $B$ ) show that the threshold current during dIPSPs $\left(V_{\text {rev }}\right.$ of $\left.-37 \mathrm{mV}\right)$ increased to $156.1 \pm$ $6.4 \%$ of control, whereas hyperpolarizing IPSPs induced smaller changes in excitability. Inhibition with $V_{\text {rev }}$ of $-37 \mathrm{mV}$ was significantly more effective than inhibition with $V_{\text {rev }}$ of $-77 \mathrm{mV}$ (two-tailed paired $t$ test; $p<$ $0.001)$, whereas the two levels of hyperpolarizing inhibition $\left(V_{\text {rev }}\right.$ of -77 and $-87 \mathrm{mV}$ ) were not different in their effect (two-tailed paired $t$ test; $p=0.596)$. PPE, Paired-pulse effect. Paired excitatory pulse protocol alone shows that part of the inhibitory effect measured during IPSPs is attributable to a relative refractory period. Current threshold for second stimulus averaged $114.7 \%$ of the current threshold for the first stimulus.

contributed to the inhibition of the second response. In pairedpulse experiments without inhibition, threshold current for the second stimulus averaged $114.7 \%$ of control $(n=5)$. This paired- 
pulse effect contributed to the total inhibition measured during the three IPSP conditions.

\section{Depolarizing IPSPs shift both the current and voltage threshold for spike generation}

Part of the inhibitory effect of dIPSPs was attributable to accommodation, a positive shift in spike threshold. Accommodation was detected as a positive shift in the absolute voltage at which spikes initiated and as an increase in the amount of depolarization required to reach threshold ( $\left.\Delta V_{\text {thresh }}\right)$. We evaluated changes in threshold for each cell by measuring the amplitude of the largest, subthreshold response to depolarizing current during control and IPSP conditions. An example recording in Figure 6 illustrates the measurement of threshold and $\Delta V_{\text {thresh }}$, under control (Fig. 6A) and dIPSP conditions (Fig. 6B). Under control conditions, 250 pA evokes the largest, subthreshold voltage response [an action potential is elicited by a $300 \mathrm{pA}$ pulse (Fig. 6A, arrow)], and $\Delta V_{\text {thresh }}$ is $13 \mathrm{mV}$. During a dIPSP induced by a synaptic conductance of $7.5 \mathrm{nS}$ (peak), current pulses ranging from 350 to 450 pA are subthreshold, and at 500 pA, only 1 in 10 trials elicits an action potential (Fig. 6B, top row). The subthreshold responses in these experiments are shown superimposed in the bottom panel of Figure $6 B$, in which five superimposed trials are shown for each level of current pulse amplitude. The voltage responses during inhibition exceeded the control threshold (Fig. 6B, solid horizontal cursor), and $\Delta V_{\text {thresh }}$ increased to $21 \mathrm{mV}$. This measure in 10 neurons revealed that dIPSPs significantly increased $\Delta V_{\text {thresh }}$ by $\sim 27 \%$, from a mean \pm SEM of $20.2 \pm 2$ to $25.6 \pm 3 \mathrm{mV}$ (twotailed paired $t$ test; $p<0.01$ ).

Shunting also contributed to the inhibitory effect of dIPSPs. To evaluate the contribution of shunting independently of the threshold shift, we compared the input resistance under control conditions and during IPSPs. Input resistance $\left(R_{\text {in }}\right)$ was measured from the slope of $I-V$ relationships. Measurements were made in the last $200 \mu \mathrm{sec}$ of the response (Fig. 6A,B, vertical cursor), and although voltage responses under these conditions were not steady state, the $I-V$ relationships appeared to be Ohmic for all cells (Fig. 6C). For the neuron in the example shown in Figure 6, a least-squares linear fit of the control data points (circles) gives an $R_{\text {in }}$ of $55 \mathrm{M} \Omega$. Measurements made during inhibition (diamonds) reveal that $R_{\text {in }}$ fell to $43 \mathrm{M} \Omega$, a change that we predicted (dashed curve) based on the resting $R_{\mathrm{in}}$ and the synaptic conductance that was applied. In nine neurons, $R_{\text {in }}$ fell from an average of $38.3 \pm 2.2 \mathrm{M} \Omega$ during control conditions to $31.6 \pm 2.8 \mathrm{M} \Omega$ during dIPSPs, a reduction of $21.2 \%$ (two-tailed paired $t$ test; $p<$ $0.05)$. This reduction was attributable to the applied inhibitory conductance; the average predicted change in $R_{\text {in }}$ was $20.8 \%$, and there was no statistically significant difference between the measured $R_{\text {in }}$ during dIPSPs and the $R_{\text {in }}$ predicted by summation of the input conductance of each cell and the applied inhibitory conductance (two-tailed paired $t$ test; $p=0.936$ ).

\section{DISCUSSION}

The primary source of GABAergic input to NM is from the SON, a brainstem nucleus receiving excitatory input from nucleus angularis and nucleus laminaris (Lachica et al., 1994; Yang et al., 1999; Monsivais et al., 2000). We suggested previously two roles for this inhibitory input in auditory processing: gain control and in regulating the fidelity of temporal encoding (Lachica et al., 1994; Yang et al., 1999; Monsivais et al., 2000). In this report, we focus on the mechanisms of GABAergic inhibition in NM neurons.
A

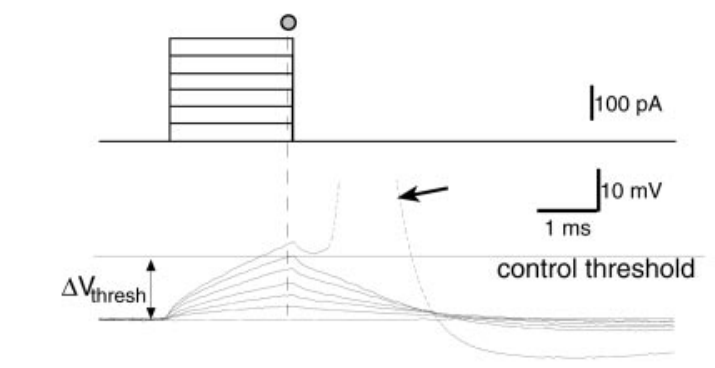

B
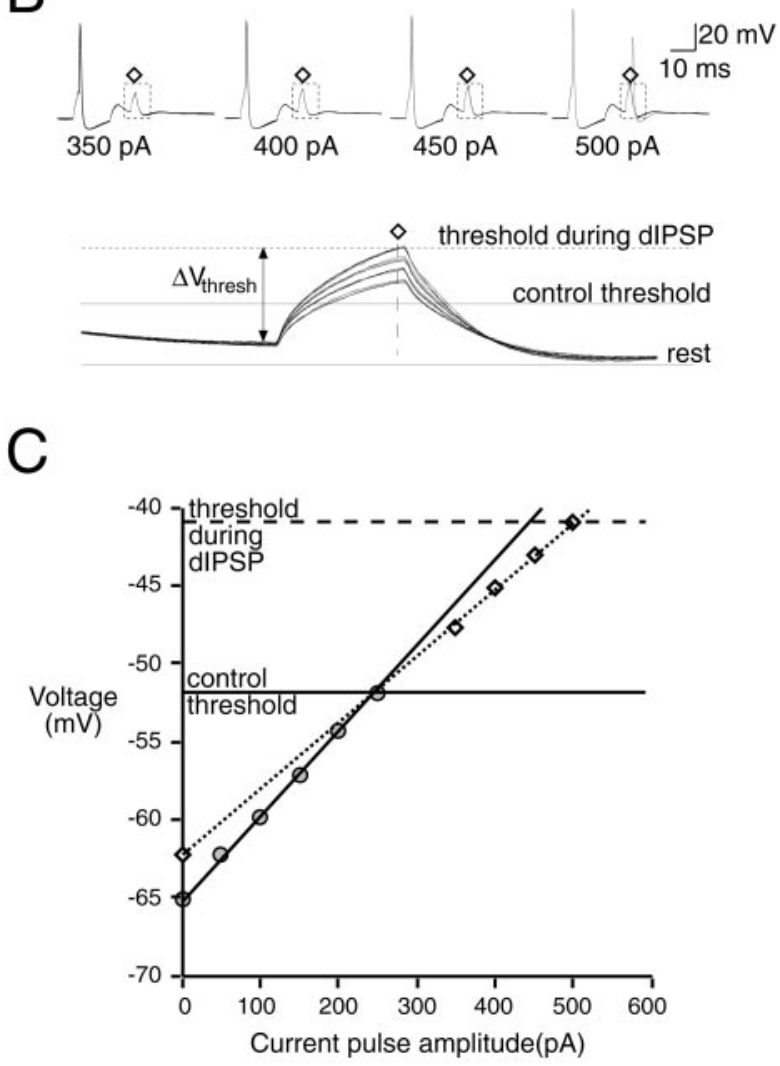

Figure 6. Accommodation contributes to the inhibitory effect of dIPSPs. $A$, Traces showing the protocol used for estimating changes in spike threshold and input resistance in an example neuron. Current pulses (top traces) applied to a cell at rest produced a family of responses (bottom traces) that provided measures of threshold (solid horizontal cursor) and $\Delta V_{\text {thresh }}$, the difference between resting membrane potential and threshold. In $B$, top traces show that there is an increase in threshold current during the mimicked IPSP, from $300 \mathrm{pA}(A)$ to $500 \mathrm{pA}$ (10 traces per current pulse level are shown). The bottom traces in $B$ are taken from these four groups of data (boxed region in each set of traces is expanded, and 5 traces are shown from each group). When current pulses coincided with a mimicked dIPSP, the threshold shifted from -52 to $-41 \mathrm{mV}$ and increased $\Delta V_{\text {thresh }}$ from 13 to $21 \mathrm{mV}$ (same scale as in $A$ ). In $C$, the $I-V$ relationship for control, subthreshold responses (circles) and responses obtained coincident with mimicked dIPSP activation (diamonds). Also, the data collected coincident with dIPSP activation (diamonds) strayed from the linear fit to control data by an amount that was predicted (dashed line) based on the summing of input conductance and the applied IPSP conductance.

GABAergic synaptic currents in NM neurons are strongly depolarizing, with a reversal potential of approximately $-36 \mathrm{mV}$. However, GABAergic synaptic potentials are not excitatory, in part because of the presence of a voltage-gated outward $\mathrm{K}^{+}$ 
conductance that counteracts the depolarizing influence of the PSPs. By mimicking natural GABAergic conductances in dynamic clamp recording, we were able to test the hypothesis that depolarizing inhibition is more effective than hyperpolarizing inhibition, which is more typical in the nervous system. For a mimicked synaptic conductance of a given amplitude, the resulting IPSP was more effective in suppressing discharges when it was depolarizing rather than hyperpolarizing. The functional advantage of dIPSPs was primarily attributable to the accommodation of spike threshold, presumably by inactivating voltage-dependent inward currents $\left(\mathrm{Na}^{+}, \mathrm{Ca}^{2+}\right.$, or a combination thereof) (Koyano et al., 1996).

Inactivation of voltage-dependent $\mathrm{Na}^{+}$currents is one factor responsible for the PAD mode of inhibition in axon terminals, in which propagating spikes are reduced in amplitude or blocked altogether (Eccles et al., 1961; Kennedy et al., 1974; Edwards, 1990; Zhang and Jackson, 1993). In modeling studies, inhibition of axon terminals was more effective when the modeled GABAergic conductance was depolarizing rather than nonpolarizing; a criterion level of inhibition could be achieved with a smaller synaptic conductance (Graham and Redman, 1994). PAD inhibition is also characterized by shunting (through GABA $_{\mathrm{A}}$ or glycine channels), and, in the experiments reported here, the shunting component of inhibition was entirely accounted for by the mimicked synaptic conductance we applied using dynamic clamp recording.

In previous reports, we described GABAergic inhibition of NM neurons and showed that both GABAergic and low-threshold $\mathrm{K}^{+}$ conductances contribute to a large shunting effect (Hyson et al., 1995; Monsivais et al., 2000). Had K ${ }^{+}$conductances contributed to the shunting in the present experiments, the observed conductance change would have been larger than what we predicted based on the sum of input conductance and synaptic conductance in each experiment. One explanation for why we found no contribution of $\mathrm{K}^{+}$conductances to shunting in the present report is that we evaluated the action of individual IPSPs, whereas the previous study measured changes in cell conductance during inhibitory plateaus induced by temporal summation of GABAergic PSPs. At high stimulation frequencies, GABAergic terminals evoke a tonic conductance in NM neurons ( $\mathrm{Lu}$ and Trussell, 2000), producing a sustained depolarization and thereby tonically activating low-threshold $\mathrm{K}^{+}$channels (Monsivais et al., 2000).

Low-threshold $\mathrm{K}^{+}$currents in $\mathrm{NM}$ neurons and other auditory centers have been implicated in regulating integration of excitatory input by minimizing EPSP duration and thereby limiting temporal summation (Reyes et al., 1994; Brew and Forsythe, 1995). These currents rapidly activate at -60 to $-70 \mathrm{mV}$, undergo little inactivation, and are blocked by either

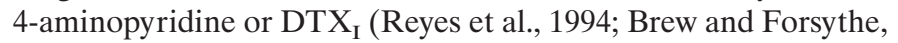

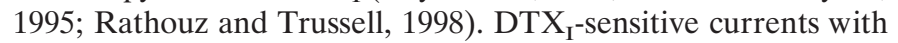
similar biophysical properties are found in dorsal root ganglion neurons (Stansfeld and Feltz, 1988) and other peripheral axons (Safronov et al., 1993) and are important in enabling accommodation in those structures (Poulter et al., 1989). Thus, a lowthreshold $\mathrm{K}^{+}$conductance may be a "prerequisite" for strongly depolarizing inhibition (e.g., PAD in axons and the similar mechanism we report here for NM neurons), because dIPSPs would otherwise elicit excitation. Our data provide the first evidence of a depolarizing, PAD-like inhibition occurring somatically in central neurons.

Several factors lead us to propose that depolarizing GABAergic input to these cells is part of their mature phenotype and not an indicator of immaturity. First, unlike depolarizing GABA or glycinergic responses in immature neurons, which are typically excitatory, GABAergic potentials in NM neurons derive much of their inhibitory efficacy by depolarizing. Second, GABAergic terminals in NM are detectable 1 full week before hatching and appear histologically mature in structure and density by hatching (Code et al., 1989). Third, hearing is nearly completely mature by hatching, based on physiological responses (Saunders et al., 1973; Rebillard and Rubel, 1981) and behavioral measures (Gray and Rubel, 1985). Finally, behavioral studies show that gallinaceous birds can localize sounds very well within $1 \mathrm{~d}$ of hatching (Gottlieb, 1965).

The recordings reported here were made in neurons from animals well after hearing onset, which occurs in ovo $\sim 10 \mathrm{~d}$ before hatching. Other studies that have shown that, in auditory neurons of mammals, responses to another inhibitory transmitter, glycine, are initially depolarizing and become hyperpolarizing during development (Kakazu et al., 1999). In these cases, however, the switch occurs before hearing onset. Consistent with this was our finding that that GABAergic currents in another auditory nucleus in the avian brainstem, the SON, reversed at more hyperpolarized potentials.

One mechanism responsible for the developmental transition from depolarizing to hyperpolarizing inhibition has been identified as $\mathrm{KCC} 2$, a K${ }^{+} / \mathrm{Cl}^{-}$cotransporter that extrudes $\mathrm{Cl}^{-}$, maintaining intracellular $\left[\mathrm{Cl}^{-}\right]$at low levels (Rivera et al., 1999). Perhaps by restricting the developmental onset of KCC2 expression, NM neurons maintain an "immature" polarity of GABAergic potentials. This mechanism may be general for classes of neurons or neuronal compartments in which low-threshold $\mathrm{K}^{+}$ currents are expressed in abundance and in which inhibition must balance particularly strong excitatory currents. Examples include $\mathrm{Na}^{+}$currents in axon terminals and large EPSCs in the somata and dendrites of neurons that are generated by large individual excitatory terminals or numerous, synchronously active inputs.

\section{REFERENCES}

Brew HM, Forsythe ID (1995) Two voltage-dependent $\mathrm{K}^{+}$conductances with complementary functions in postsynaptic integration at a central auditory synapse. J Neurosci 15:8011-8022.

Cattaert D, El Manira A (1999) Shunting versus inactivation: analysis of the presynaptic inhibitory mechanisms in primary afferents of the crayfish. J Neurosci 19:6079-6089.

Code RA, Burd GD, Rubel EW (1989) Development of GABA immunoreactivity in brainstem auditory nuclei of the chick: ontogeny of gradients in terminal staining. J Comp Neurol 284:504-518.

Coombs JS, Eccles JC, Fatt P (1955) The inhibitory suppression of reflex discharges from motoneurons. J Physiol (Lond) 130:396-413.

Eccles JC, Eccles RM, Magni F (1961) Central inhibitory action attributable to presynaptic depolarization produced by muscle afferent volleys. J Physiol (Lond) 159:147-166.

Edwards DH (1990) Mechanisms of depolarizing inhibition at the crayfish giant motor synapse. I. Electrophysiology. J Neurophysiol 64:532-540.

Ehrlich I, Löhrke S, Friauf E (1999) Shift from depolarizing to hyperpolarizing glycine action in rat auditory neurons is due to agedependent $\mathrm{Cl}^{-}$regulation. J Physiol (Lond) 520:121-137.

Gottlieb G (1965) Imprinting in relation to parental and species identification by avian neonates. J Comp Physiol Psychol 59:345-356.

Graham B, Redman S (1994) A simulation of action-potentials in synaptic boutons during presynaptic inhibition. J Neurophysiol 71:538-549.

Gray L, Rubel EW (1985) Development of absolute thresholds in chickens. J Acoust Soc Am 77:1162-1172.

Hopkins WF, Allen ML, Houamed KM, Tempel BL (1994) Properties of voltage-gated $\mathrm{K}$ currents expressed in Xenopus oocytes by mKv1.1, mKv1.2 and their heteromultimers as revealed by mutagenesis of the dendrotoxin-binding site in mKv1.1. Pflügers Arch 428:382-390.

Hyson RL, Reyes AD, Rubel EW (1995) A depolarizing inhibitory response to GABA in brainstem auditory neurons of the chick. Brain Res 677:117-126. 
Kakazu Y, Akaike N, Komiyama S, Nabekura J (1999) Regulation of intracellular chloride by cotransporters in developing lateral superior olive neurons. J Neurosci 19:2843-2851.

Kennedy D, Calabrese RL, Wine JJ (1974) Presynaptic inhibition: primary afferent depolarization in crayfish neurons. Science 186:451-454.

Koyano K, Funabiki K, Ohmori H (1996) Voltage-gated ionic currents and their roles in timing coding in auditory neurons of the nucleus magnocellularis of the chick. Neurosci Res 26:29-45.

Kyrozis A, Reichling DB (1995) Perforated-patch recording with gramicidin avoids artifactual changes in intracellular chloride concentration. J Neurosci Methods 57:27-35.

Lachica EA, Rübsamen R, Rubel EW (1994) GABAergic terminals in nucleus magnocellularis and nucleus laminaris originate from the superior olivary nucleus. J Comp Neurol 348:403-418.

Lu T, Trussell LO (2000) Inhibitory transmission mediated by asynchronous transmitter release. Neuron 26:683-694.

Monsivais P, Yang L, Rubel EW (2000) GABAergic inhibition in nucleus magnocellularis: implications for phase locking in the avian auditory brainstem. J Neurosci 20:2954-2963.

Owens DF, Boyce LH, David MBE, Kriegstein AR (1996) Excitatory GABA responses in embryonic and neocortical slices demonstrated by gramicidin perforated-patch recordings and calcium imaging. J Neurosci 16:6414-6423.

Poulter MO, Hashiguchi T, Padjen AL (1989) Dendrotoxin blocks accommodation in frog myelinated axons. J Neurophysiol 62:174-184.

Qian N, Sejnowski TJ (1990) When is an inhibitory synapse effective? Proc Natl Acad Sci USA 87:8145-8149.

Rathouz M, Trussell LO (1998) Characterization of outward currents in neurons of the avian nucleus magnocellularis. J Neurophysiol 80:2824-2835.

Rebillard G, Rubel EW (1981) Electrophysiological study of the maturation of auditory responses from the inner ear of the chick. Brain Res 229:15-23.

Reyes AD, Rubel EW, Spain WJ (1994) Membrane properties underlying the firing of neurons in the avian cochlear nucleus. J Neurosci 14:5352-5364.
Rivera C, Voipio J, Payne JA, Ruusuvuori E, Lahtinen H, Lamsa K, Privola U, Saarma M, Kaila K (1999) The $\mathrm{K}^{+} / \mathrm{Cl}^{-}$co-transporter $\mathrm{KCC} 2$ renders GABA hyperpolarizing during neuronal maturation. Nature 397:251-255.

Robertson B, Owen DG (1992) Pharmacology of a cloned potassium channel from mouse brain (MK-1) expressed in CHO cells: effects of blockers and an "inactivation peptide." Br J Pharmacol 109:725-735.

Robinson HPC, Kawai N (1993) Injection of digitally synthesized synaptic conductance transients to measure the integrative properties of neurons. J Neurosci Methods 49:157-165.

Rudomin P, Schmidt RF (1999) Presynaptic inhibition in the vertebrate spinal cord revisited. Exp Brain Res 129:1-37.

Safronov BV, Kampe K, Vogel W (1993) Single voltage-dependent potassium channels in rat peripheral nerve membrane. J Physiol (Lond) 460:675-691.

Saunders JC, Cole RB, Gates GR (1973) The development of auditory evoked responses in the cochlea and cochlear nuclei of the chick. Brain Res 63:59-74.

Sharp AA, O'Neil MB, Abbott LF, Marder E (1993) Dynamic clamp: computer-generated conductances in real neurons. J Neurophysiol 69:992-995.

Smith TG, Wuerker RB, Frank K (1967) Membrane impedance changes during synaptic transmission in cat spinal motoneurons. J Neurophysiol 30:1072-1096.

Staley KJ, Mody I (1995) Shunting of excitatory input to dentate gyrus granule cells by a depolarizing $\mathrm{GABA}_{\mathrm{A}}$ receptor-mediated postsynaptic conductance. J Neurophysiol 68:197-212.

Stansfeld C, Feltz A (1988) Dendrotoxin-sensitive K channels in dorsal root ganglion cells. Neurosci Lett 93:49-55.

Yang L, Monsivais P, Rubel EW (1999) The superior olivary nucleus and its influence on nucleus laminaris: a source of inhibitory feedback for coincidence detection in the avian auditory brainstem. J Neurosci 19:2313-2325.

Zhang SJ, Jackson MB (1993) GABA-activated chloride channels in secretory nerve endings. Science 259:531-534. 